\title{
Le discours « interjectionnel » dans la linguistique russe après 1950 : les origines des grammaires académiques
}

\section{Ekaterina Velmezova}

\section{CpenEdition}

\section{Journals}

Édition électronique

URL : http://journals.openedition.org/edl/321

DOI : $10.4000 /$ edl.321

ISSN : 2296-5084

Éditeur

Université de Lausanne

\section{Édition imprimée}

Date de publication : 15 décembre 2009

Pagination : 151-166

ISBN : 978-2-940331-21-5

ISSN : 0014-2026

\section{Référence électronique}

Ekaterina Velmezova, «Le discours « interjectionnel » dans la linguistique russe après 1950 : les origines des grammaires académiques », Études de lettres [En ligne], 4 | 2009, mis en ligne le 15 décembre 2012, consulté le 18 décembre 2020. URL : http://journals.openedition.org/edl/321 ; DOI : https://doi.org/10.4000/edl.321 


\section{LE DISCOURS "INTERJECTIONNEL" DANS LA LINGUISTIQUE RUSSE APRÈS 1950: LES ORIGINES DES GRAMMAIRES ACADÉMIQUES}

Le discours «interjectionnel» en linguistique soviétique de la deuxième moitié du $\mathrm{XX}^{\mathrm{e}}$ siècle est étudié dans cet article à travers l'analyse de trois grammaires du russe dites "académiques» qui datent de 1952-1954, 1960 et 1980. Tandis que, dans ces grammaires, l'influence des théories linguistiques de V.V. Vinogradov est incontestable dans la description des autres parties du discours, la situation de l'étude des interjections semble plus compliquée, voire paradoxale: tout en s'appuyant sur la définition de l'interjection proposée par Vinogradov, certains auteurs de grammaires académiques manifestent un écart considérable par rapport à son approche de l'étude de ces mots. Le rôle de Vinogradov dans l'étude de la morphologie russe en général est également discuté dans l'article.

En discutant des rapports entre la linguistique et les changements sociaux, il ne serait pas exagéré d'affirmer que, dans l'histoire de la linguistique soviétique, l'événement le plus important fut l'intervention de Staline dans les discussions sur la langue en 1950. Sans vouloir analyser le discours du dictateur soviétique dans cet article ${ }^{1}$, voyons comment l'intervention de Staline a pu influencer, de façon indirecte bien sûr, l'étude des interjections en URSS. Comme nous l'avons montré dans un article consacré au discours «interjectionnel» dans la linguistique russe et soviétique durant tout le $\mathrm{XX}^{\mathrm{e}}$ siècle ${ }^{2}$, le champ même des recherches

I. D’autant plus que cela a déjà été fait maintes fois, y compris dans nos propres travaux (cf. par exemple E. Velmezova, «La sémantique idéologique entre Marr et Staline» et $i d$. ., Les lois $d u$ sens, p. 225-230).

2. E. Velmezova, «L'interjection dans la linguistique russe du $\mathrm{XX}^{\mathrm{e}}$ siècle». 
correspondantes s'est considérablement rétréci après 1950 : l'intervention de Staline en linguistique soviétique pourrait être, en quelque sorte, comparée à la première résolution de la Société linguistique de Paris en 1866, car ces événements ont mis un terme, qui semblait définitif, à la réflexion sur les origines du langage. En conséquence, après l'intervention de Staline, les interjections n'étaient plus considérées comme les éléments primaires du langage humain - comme c'était souvent le cas auparavant. Ainsi en URSS la problématique langagière de leur étude a cédé la place à une problématique purement linguistique.

Actuellement, en Russie, les interjections peuvent être étudiées sous plusieurs aspects: fonctionnel et communicatif, comparatif (supposant une analyse des interjections dans plusieurs langues, apparentées ou non apparentées) ${ }^{3}$, lié aux problèmes des langues en contacts (entre autres, on y fait souvent attention aux interjections que le russe emprunte aux autres langues), etc. En même temps, en ce qui concerne la «tradition» linguistique académique russe, c'est toujours la dernière grammaire dite académique de la langue russe, éditée encore en $1980^{4}$, qui sert de base "officielle» pour la description des interjections (comme, d'ailleurs, à quelques exceptions près, pour l'étude des autres parties du discours). Aujourd'hui, quand la nécessité de créer une nouvelle grammaire académique du russe devient de plus en plus manifeste, nous avons la possibilité d'étudier les grammaires académiques déjà existantes ${ }^{5}$ non seulement pour mieux apprécier leurs qualités et déceler leurs défauts, mais aussi pour essayer de comprendre quelles en étaient les origines théoriques, explicites ou non. Comment les interjections sont-elles analysées dans les trois grammaires académiques? ${ }^{6}$

3. Ibid., point 2.2.

4. N.Ju. Švedova et al., Russkaja grammatika.

5. A part la grammaire russe de 1980 qui a déjà été mentionnée, les deux autres grammaires académiques de la langue russe sont: V.V. Vinogradov et al., Grammatika russkogo jazyka (1952-1954) et V.V. Vinogradov et al., Grammatika russkogo jazyka (1960).

6. Dans les deux premières grammaires académiques, les parties consacrées aux interjections ont été composées par N.Ju. Švedova (qui est l'auteur des autres études importantes consacrées aux interjections, cf. par exemple N.Ju. Švedova, «Meždometija kak grammatičeski značimyj èlement predloženija v russkoj razgovornoj reči», etc. [sur l'importance de cet article pour le discours «interjectionnel» en Russie, cf. E. Velmezova, "L'interjection dans la linguistique russe du XX $\mathrm{XX}^{\mathrm{e}}$ siècle", point 1.1]); dans la grammaire académique de 1980, l'auteur de la partie correspondante fut I.N. Kručinina (par la suite, elle écrira l’article «Interjections» [Meždometija] 
En général, avec le temps, dans ces trois grammaires, ainsi que dans d'autres descriptions grammaticales du russe qui servaient de références "officielles" (par exemple, pour la préparation des thèses de doctorat [kandidatskaja dissertacija] ou d'habilitation [doktorskaja dissertacija], etc.) ${ }^{7}$, l'interprétation de la notion d'«interjection» changeait peu. Les auteurs de ces travaux considéraient les interjections comme une partie du discours à part, par rapport aux mots lexicaux [samostojatel'nye (ou znamenatel'nye) časti reči i] et grammaticaux [služebnye časti reči]; ils distinguaient en même temps deux groupes sémantiques d'interjections: les interjections qui expriment 1) les sentiments et 2) la volonté. Seule pouvait changer, par rapport aux interjections, la position des mots qui étaient grammaticalement "problématiques» (comme les onomatopées, les interjections verbales, les mots da 'oui' et net 'non' ${ }^{8}$, etc.).

Ainsi deux questions surgissent:

1) Qui dans la linguistique soviétique est à l'origine de la distinction des interjections en tant que partie du discours «à part", qui n'adhère ni aux mots lexicaux, ni aux mots grammaticaux?

2) D'où vient la division des interjections précisément en deux groupes?

D'après N.S. Pospelov, la première grammaire académique du russe (c'est-à-dire la grammaire qui, en quelque sorte, a servi de base et de modèle pour les autres grammaires académiques) s'appuie en grande partie sur la "théorie grammaticale du mot» de V.V. Vinogradov ${ }^{9}$, développée dans son livre de 1947 Langue russe. Théorie grammaticale du mot [Russkij jazyk. Grammatičeskoe učenie o slove ${ }^{10}$. Jusqu'à quel point cette thèse est-elle exacte par rapport à la façon dont les interjections y sont présentées?

pour le Dictionnaire encyclopédique linguistique [Lingvističeskij ènciklopedičeskij slovar'], cf. I.N. Kručinina, «Meždometija»).

7. A.N. Gvozdev, Sovremennyj russkij literaturnyj jazyk, p. 423-427; N.Ju. Švedova, Grammatika sovremennogo russkogo literaturnogo jazyka; «Meždometie»; N.Ju. Švedova, "Meždometija», etc.

8. Sur le rattachement des mots da et net tantôt aux interjections, tantôt aux particules, cf. E. Velmezova, «Da i net: časticy ili meždometija?».

9. N.S. Pospelov, Učenie o častjax reči v russkoj grammatičeskoj tradicii. Materialy $k$ kursam jazykoznanija, p. 35, 45.

Io. Cf. V.V. Vinogradov, Russkij jazyk. La base même du livre de Vinogradov est constituée par la deuxième partie de son travail de 1938 (cf. V.V. Vinogradov, Sovremennyj russkij jazyk). 
En interprétant le mot [slovo] comme une unité des significations lexicales et grammaticales, Vinogradov distingue quatre types (quatre catégories sémantico-structurelles) de mots russes: 1) les parties du discours; 2) les particules du discours; 3) les mots et particules modaux; 4) les interjections ${ }^{11}$. Ainsi les interjections sont considérées ici comme des mots à part, ce qui correspond à leur place dans la première grammaire académique, et cela à une exception près: chez Vinogradov, les interjections ne font pas partie de ce qu'il désigne comme parties du discours en tant que telles. D'ailleurs, d'après Vinogradov, si on discute des anciennes classifications des parties du discours en russe (dans lesquelles, habituellement, dix parties du discours [y compris les interjections] sont distinguées ${ }^{12}$ ), parmi ces dernières il y en aurait de très problématiques, et c'est précisément le «droit» de l'interjection d'être désignée comme partie du discours à part entière qui pourrait être contesté le plus facilement. En citant sous ce rapport J. Vendryes, Vinogradov accepte le point de vue du linguiste français, selon qui

[...] quelle que soit l'importance de l'interjection dans l'usage, elle a en soi quelque chose qui la met à part des autres parties du discours, elle ne peut être rangée dans un même classement. [...] Elle n’a en général rien à faire avec la morphologie. Elle représente une forme spéciale du langage, le langage affectif, ou parfois le langage actif; en tout cas, elle reste en dehors de la structure du langage intellectuel ${ }^{13}$.

En général, la classification des mots russes proposée par Vinogradov était très novatrice pour la "tradition" académique russe: Vinogradov aurait réussi, en quelque sorte, à «réconcilier» deux approches différentes de la description de la morphologie russe, qui coexistaient en Russie au début du XX ${ }^{\mathrm{e}}$ siècle ${ }^{14}$. La première approche, dite "formelle», remontait à l'école de F.F. Fortunatov: c'est la forme des mots qui y servirait de critère principal pour distinguer les parties du discours. La deuxième approche, qui était propre aux travaux d'I.A. Baudoin de Courtenay

II. V.V. Vinogradov, Russkij jazyk, p. 22, 35, 45.

I2. Ou, écrit Vinogradov, si l'on considère (comme A.A. Potebnja, F.F. Fortunatov et A.M. Peškovskij), que les pronoms et les numéraux ne constituent pas des parties du discours à part entière, il nous faudra nous limiter à huit parties du discours.

I3. J. Vendryes, Le langage, p. 134. Vinogradov se réfère à la traduction russe de ce livre datant de 1937 (Ž. Vandries, Jazyk).

I4. Cf. par exemple Ju.A. Bel'čikov, Akademik V.V. Vinogradov, p. 46 sq. 
et L.V. Ščerba, est parfois désignée comme "fonctionnelle» ${ }^{15}:$ la grammaire y était considérée

[...] non pas comme une théorie des formes [učenie o formax], mais comme un système compliqué de correspondances entre les sens $[$ smysly] qui constituent le contenu de la parole, et les formes externes de l'expression de ces sens, de leurs caractéristiques formelles [formal'nye pokazateli] ${ }^{16}$.

Le fait que Vinogradov ait réussi à réunir ces deux approches dans son travail semble, à première vue, trouver confirmation dans sa description des interjections: pour prouver cette thèse, il suffit de regarder comment les interjections avaient été interprétées par Fortunatov et Ščerba.

A la base de l'approche «formelle» du problème de la distinction des parties du discours, qui était propre aux descriptions linguistiques de Fortunatov et de ses élèves, se trouve la notion de "mot isolé» [otdel'noe slovo], défini par Fortunatov comme

[...] un son de la parole ou un ensemble [kompleks] des sons de la parole qui possède, dans la langue, un sens indépendamment des autres sons [...] et qui, s'il s'agit d'un ensemble de sons, ne peut être divisé en mots isolés sans que le sens de l'une ou de l'autre partie de cet ensemble phonétique ne soit perdu ${ }^{17}$.

Fortunatov établit ensuite une distinction entre les mots à part entière [polnye slova], les mots partiels [častičnye slova] et les interjections, en se basant sur des critères syntaxiques et sémantiques ${ }^{18}$. Ainsi, les mots à

I5. Ibid., p. 47.

I6. L.R. Zinder, Ju.S. Maslov, L.V. Ščerba, p. 69. Cf. aussi le point de vue d'I.P. Ivanova, selon qui Ščerba distinguait les parties du discours en se basant sur les paramètres suivants: "la sémantique lexicale, les catégories morphologiques et le fonctionnement syntaxique» (I.P. Ivanova, «O polevoj strukture častej reči v anglijskom jazyke», p. 125). Néanmoins, à l'encontre d'une opinion généralement répandue, Fortunatov s'appuyait lui aussi sur des critères sémantiques et syntaxiques quand il parlait des parties du discours (cf. F.F. Fortunatov, Sravnitel'noe jazykovedenie, p. 131 et suiv.), cf. aussi plus loin.

I7. F.F. Fortunatov, Sravnitel'noe jazykovedenie, p. 169. Vinogradov critiquait cette formulation en la considérant comme tautologique, car, en essayant de définir le mot, Fortunatov se serait appuyé sur cette même notion (cf. V.V. Vinogradov, Sovremennyj russkij jazyk, p. 38).

I8. Cf. aussi ibid., p. 42. 
part entière «désignent les objets de la pensée et, par rapport aux propositions [predloženija], constituent soit leurs parties, soit des propositions entières» ${ }^{19}$. Quant aux mots partiels, leurs sens [značenija] "n'existent pas indépendamment des sens des mots à part entière, car les mots partiels désignent quelque chose soit dans la sémantique [značenie] des mots à part entière, soit dans la sémantique [značenie] des propositions dont ils font partie» ${ }^{20}$. Enfin, les interjections sont les mots qui, premièrement, soit existent en dehors des propositions, soit constituent des propositions entières, et, deuxièmement, n'expriment pas des idées, mais des sentiments ${ }^{21}$.

En ce qui concerne la deuxième approche de la distinction des parties du discours (qui était manifeste, entre autres, dans les travaux de Ščerba), l'intérêt même de ce linguiste pour les interjections était ambivalent: Ščerba étudiait les interjections du point de vue tantôt diachronique

19. F.F. Fortunatov, Sravnitel'noe jazykovedenie, p. 134.

20. Ibid., p. 169.

2I. Ibid., p. 171. Vinogradov avait tort d'affirmer que l'absence de «forme» (sur la définition de «forme» chez Fortunatov, cf. plus loin) servait aussi de critère à Fortunatov pour distinguer les interjections (V.V. Vinogradov, "Učenie akad. A.A. Šaxmatova o grammatičeskix formax slov i o častjax reči v sovremennom russkom jazyke», p. 9): d'ailleurs, il se corrige plus tard, à la même page. Fortunatov développe ensuite la thèse de la nécessité de distinguer, d'un côté, les cris instinctifs et, de l'autre, les interjections comme les mots de la langue: c'est la volonté ( $v$ s. son absence) de celui qui parle qui sert à distinguer les deux (F.F. Fortunatov, Sravnitel'noe jazykovedenie, p. 172). Soulignons qu'en parlant des interjections, Fortunatov ne donne pratiquement pas d'exemples: les seuls exemples qu'on trouve chez lui concernent l'interaction des interjections avec les mots à part entière. Fortunatov écrit ainsi : «[...] dans la phrase on voskliknul ax! ['Ah, cria-t-il'] le mot $a x$ 'ah' n'est plus une interjection, mais un mot à part entière qui signifie l'interjection $a x$. C'est pourquoi, à partir des interjections peuvent être dérivées les bases de mots à part entière qui possèdent des formes, comme, par exemple, axat' 'pousser des ah' ou oxat' 'pousser des oh' en russe. D'autre part, les mots à part entière peuvent eux aussi être utilisés dans la langue comme des interjections, comme par exemple en russe bože, bože moj 'mon Dieu', čert 'diable'" (ibid.). A part la notion de «mot isolé», c'est la notion de «forme du mot» [forma slova] qui était importante pour Fortunatov. A la différence de Potebnja, qui interprétait la notion de «forme (interne)» du point de vue lexicologique et sémantique (sur l'évolution de la notion de «forme interne» chez Potebnja, cf. M.G. Jaroševskij, «Ponjatie vnutrennej formy»), Fortunatov définissait la forme du mot comme la propriété [sposobnost'] des mots particuliers de se diviser, dans la conscience des locuteurs, en partie de base et en partie formelle [formal'naja i osnovnaja prinadležnost'slova] (F.F. Fortunatov, Sravnitel'noe jazykovedenie, p. 136). C'est à la base de cette notion que Fortunatov divise les mots à part entière en plusieurs classes grammaticales (les substantifs, les verbes, etc.). 
(voire "préhistorique»), tantôt synchronique ${ }^{22}$. Mais, si son analyse «diachronique» des interjections, considérées comme des «sons [préhistoriques] diffus ${ }^{23}$, est restée relativement peu connue des linguistes soviétiques, le travail de Ščerba sur les parties du discours en russe ${ }^{24}$ a, en revanche, eu une grande résonance dans la linguistique russe. La première chose que Ščerba fait en abordant l'étude des parties du discours en russe, c'est de distinguer la catégorie "très vague et peu claire» ${ }^{25}$ des interjections qui exprimeraient des émotions et ne posséderaient aucun élément cognitif. L'autre critère pour distinguer les interjections chez Ščerba est le critère syntaxique - comme chez Fortunatov. Ainsi, pour Ščerba, les interjections sont syntaxiquement isolées, et n'ont pas de liens avec d'autres éléments dans la parole [otsutstvie svjazej (...) v potoke reči ${ }^{26}$. Même si Ščerba ne le formule pas explicitement, il distingue dans son article les parties du discours (qui seraient pour lui des objets réels plutôt que des objets de connaissance) à l'aide de critères psycholinguistiques: dans cette partie de sa conception, il semble très influencé non seulement par son maître Baudoin de Courtenay ${ }^{27}$, mais aussi par la

22. La conception «interjectionnelle» de Ščerba est analysée en détail dans notre article: E. Velmezova, «Eléments primaires du langage humain ou catégories psycholinguistiques propres aux langues? Les interjections chez L.V. Ščerba”.

23. L.V. Ščerba, "O "diffuznyx zvukax" ".

24. L.V. Ščerba, "O častjax reči v russkom jazyke».

25. Ibid., p. 81.

26. Ibid., p. 81 sq. A la base de ces deux critères (sémantique et syntaxique) de distinction, les interjections sont également définies dans la grammaire scolaire du russe de S.G. Barxudarov, éditée par Ščerba: «Les interjections servent à exprimer les émotions qui accompagnent la pensée énoncée. [...] les interjections ne font pas partie des membres de la proposition [členy predloženija]» (S.G. Barxudarov, Grammatika russkogo jazyka, partie II, p. 65).

27. Cf., par exemple, la référence de Baudoin de Courtenay à des travaux de V.A. Bogorodickij: d'après Baudoin, Bogorodickij "avait tout à fait raison» de considérer les parties du discours comme des "catégories réelles de notre esprit [dejstvitel'nye kategorii našego uma]" (I.A. Boduèn de Kurtenè, "Lingvističeskie zametki i aforizmy», p. 47); ailleurs, Baudoin de Courtenay mentionne une «distinction des parties du discours qui correspondent à des critères psychiques objectifs [ob"ektivno-psixičeskoe različenie častej reči i]» (I.A. Boduèn de Kurtenè, "Jazyk i jazyki», p. 79). En se référant, encore une fois, à Bogorodickij, Baudoin de Courtenay mentionne les « "interjections" et leur type particulier - les “onomatopées” qui étaient, “à l’origine, une sorte des réflexes perçus par l'oreille et reproduits par les organes de la parole [refleksy so sluxa na organ proiznošenija]" " (I.A. Boduèn de Kurtenè, "Lingvističeskie zametki i aforizmy", p. 48). (D'après Baudoin de Courtenay, en parlant des interjections, Bogorodickij 
Philosophie de la grammaire d'O. Jespersen. Le fait que Ščerba désigne la catégorie des interjections russes comme floue et imprécise devrait indiquer que c'est d'une façon vague et imprécise que les mots de ce groupe sont représentés par tous les russophones ${ }^{28}$.

Comme on peut le voir, même si Vinogradov aurait, en ce qui concerne le problème de la typologie des parties du discours en général, réconcilié les deux traditions descriptives différentes de la morphologie russe, par contre, pour ce qui est de la distinction des interjections comme un groupe particulier par rapport à d'autres mots de la langue russe ${ }^{29}$, Vinogradov a pu s'appuyer sur ces deux traditions simultanément: les approches de Fortunatov et de Ščerba de la distinction des autres parties du discours n'étaient pas les mêmes, mais les deux chercheurs, comme aussi bien d'autres ${ }^{30}$, considéraient les interjections comme des mots à part, par rapport à d'autres parties du discours et se basaient sur les mêmes critères - sémantique et syntaxique. L'innovation de Vinogradov par rapport aux approches des interjections qu'on trouve chez Fortunatov et chez Ščerba consiste en particulier dans le fait qu'il propose des critères plus nombreux pour distinguer les interjections en tant que groupe de mots à part: 1) l'intonation; 2) l'absence de côté cognitif dans leur sémantique (les interjections «expriment les émotions, les états d'âme et la volonté du sujet, sans les désigner, sans les nommer» $\left.{ }^{31}\right)$; 3) leur "absence d'organisation» [neorganizovannost'] syntaxique ( la question de savoir si les interjections peuvent ou non

aurait dû mentionner également ce que I.I. Sreznevskij désignait comme "particules verbales [glagol'nye časticy]» [ibid.]).

28. D'ailleurs, Jespersen ne distinguait même pas les interjections en tant que partie du discours à part entière (O. Jespersen, La philosophie de la grammaire, p. 110 $s q$.). Sur les différences théoriques entre les approches de Ščerba et de Jespersen, cf. E. Velmezova, «Eléments primaires du langage humain ou catégories psycholinguistiques propres aux langues?», p. 218 sq.

29. Cf. la distinction ultérieure (adoptée, entre autres, dans les grammaires académiques) des interjections dans un groupe particulier de mots qui n'appartiennent ni aux mots lexicaux, ni aux mots grammaticaux - ce à quoi nous avons déjà fait attention au début de cet article.

30. Cf. la thèse suivante de Vinogradov: "Parallèlement à la distinction des mots russes en ces deux catégoriques générales [il s’agit de la distinction des mots lexicaux et des mots grammaticaux $-E$. $V$.], depuis longtemps, les chercheurs traçaient les bases pour la distinction d'une troisième catégorie [issledovateljami namečalas' tret' ja kategorija] - celle des interjections" (V.V. Vinogradov, Russkij jazyk, p. 34).

3I. Ibid., p. 35. 
constituer des propositions reste discutable» $\left.{ }^{32}\right)$; 4) l'absence de forme morphologique [morfologičeskaja nedelimost'] ; 5) leur côté affectif; 6) leur lien direct avec la mimique et le geste expressif (les interjections «se rapprochent plutôt des gestes expressifs que des mots-désignations [slova-nazvanija]" $\left.{ }^{33}\right)$.

En ce qui concerne la question de la distinction des deux groupes d'interjections, l'une des différences principales dans la description des interjections chez Vinogradov et dans la première grammaire académique consiste en ce que seuls deux groupes d'interjections (cf. plus haut) sont distingués dans cette dernière étude. Cela pourrait être considéré en quelque sorte comme un écart important aux principes de l'approche de Vinogradov de l'étude des interjections, comme un retour aux anciennes classifications des interjections, quand, d'après Vinogradov, "les groupes principaux d'interjections n'étaient pas encore distingués (à part la division des interjections en émotionnelles, impératives et onomatopéiques) " ${ }^{34}$. En même temps, la contribution particulière de Vinogradov à l'étude des interjections consistait, tout d'abord, dans le fait qu'il distinguait dix (!) «catégories sémantico-grammaticales» [semantiko-grammatičeskie razrjady] d'interjections ${ }^{35}$ :

1) les interjections (non dérivées des mots lexicaux ${ }^{36}$ ) qui expriment les sentiments, comme $a$ ! 'ha!', $a x$ ! 'ah!', ba!' oh!' (pour exprimer l'étonnement), aj da!' ah!' [par exemple, dans la phrase Aj da molodec! 'Ah, le gaillard!'. - E. V. ], ura!' hourra!' (pour exprimer l'approbation),

32. Ibid.

33. Ibid.

34. Ibid., p. 616.

35. Cf. aussi l'expression de Vinogradov «la différentiation grammatico-sématique à l'intérieur des interjections [grammatiko-semantičeskaja differenciacija vnutri meždometij]»(V.V. Vinogradov, Russkij jazyk, p. 46). Sous ce rapport, Vinogradov avait raison de reprocher aux grammairiens russes des époques précédentes une absence d'intérêt pour la classification des interjections. Même A.A. Šaxmatov, qui, d'après Vinogradov, "avait introduit une certaine clarté dans l'étude des interjections» (ibid., p. 613, cf. aussi V.V. Vinogradov, Sovremennyj russkij jazyk, p. 570), ne prenait pas le problème de la classification des interjections suffisamment au sérieux (V.V. Vinogradov, Russkij jazyk, p. 616; cf. aussi l'analyse de l'étude des interjections chez Šaxmatov dans le même livre de Vinogradov, p. 613-615).

36. V.V. Vinogradov, Russkij jazyk, p. 619. 
spasibo 'merci' (pour exprimer la gratitude) ${ }^{37}$, to-to (pour exprimer la satisfaction mélangée avec une menace ${ }^{38}$ [comme, par exemple, dans les phrases To-to on budet rad 'C'est là qu'il sera content'; To-to i ono 'C'est bien ça' - E. V.]), užo: vot užo (pour exprimer la menace), è! 'hé!', èx! 'ah!', i!, nu-už! 'ah!' (pour exprimer le reproche), t'fu! 'pouah!', fi!, u!, a!, fu! 'fi!' (pour exprimer le dégoût), uf! 'ouf!' (pour exprimer la fatigue), uh!'ah!' (pour exprimer l'effroi), oj-oj!, uu!' oh!' (pour exprimer la peur, la crainte, la douleur), o!, uvy! 'hélas!' (pour exprimer la tristesse, le chagrin), etc.;

2) les interjections (dérivées des mots lexicaux ${ }^{39}$ ) qui expriment des émotions, ainsi batjuški!' mes chers pères!', gluposti! 'quelle bêtise!', strast'!, užas!' quelle horreur!', beda!, gore!' quel malheur!', etc.;

3) les interjections qui "expriment une caractéristique émotionnelle ou une appréciation de l'état des choses, plutôt qu'une expression spontanée d'une émotion, d'un état d'esprit et d'un sentiment " ${ }^{40}$ - par exemple, delo - tabak! 'l'affaire est tombée à l'eau!', kryška! kajuk! kaput!'c'en est fait!', 'l'affaire est fichue!', plevoe delo!'ce n'est rien du tout!', f' juit'! [comme par exemple dans l'expression on f'juit'! 'il a filé!', 'il a pris la clef des champs!' - E. V.], etc.;

4) les interjections qui expriment la volonté, comme von! 'hors d'ici!', proč!!, brys!' 'ouste!', doloj! 'bas!', 'à bas!', polno! 'assez!' (les interjections qui servent à exprimer "l'éloignement" [otčuždenie]), nu!'eh bien!', 'allons!' (pour exprimer l'impulsion), polno-polnote!' allons donc!', 'voyons!' (pour exprimer la conviction'), stop! 'halte!', 'stop!', no! [pour exprimer un désaccord teinté de menace. - E. V.], cyc!, ts!, ‘šs! 'chut!' (pour exprimer l'interdiction [de parler. $-E . V$.$] );$

5) les interjections qui expriment une attitude émotionnelle et "volontaire» de l'interlocuteur, ainsi da! 'oui!', konečno! 'bien sûr!' (pour exprimer la confirmation), net!' non!' (pour exprimer le refus), vot ešče!

37. Il serait plus logique de rattacher cette interjection au groupe suivant ou au groupe numéro six - où, d'ailleurs, nous la trouvons plus tard (cf. infra). Cet exemple, ainsi que d'autres montrent que la classification proposée par Vinogradov n'est pas irréprochable d'un point de vue logique.

38. Aujourd'hui, il est difficile de distinguer une «menace» dans la sémantique du mot to-to: d'ailleurs, la sémantique de certaines autres interjections que Vinogradov donne comme exemples a aussi changé avec le temps.

39. V.V. Vinogradov, Russkij jazyk, p. 619.

40. Ibid., p. 619 sq. 
'par exemple!', pravo!' allons donc!' (pour exprimer l'assurance [uverenie]), a? 'comment?', as'? 'hein?', čto? 'quoi ?' (pour répondre), oj li? 'est-ce possible?' (pour exprimer la méfiance), etc.;

6) les interjections de l'étiquette, comme mersi!, spasibo!, blagodarstvujte! (un mot vieilli) 'merci!', zdras'te!' bonjour!', izvinjajus'‘ pardon', etc.;

7) les interjections injurieuses, par exemple, čert voz'mi! čert poberi! 'ah!', 'diable!', 'sapristi!', etc.;

8) les interjections vocatives, comme gospodi moj!' Seigneur!';

9) les exclamations onomatopéiques (qui «touchent aux interjections» ${ }^{41}$, ainsi bac! 'pan!', xlop! 'vlan!', bux!'pan!', 'pouf!', 'patatras!');

10) les "formes "interjectionnelles" verbales" qui se trouvent entre les verbes et les interjections, comme, par exemple, xlop 'pan!', 'vlan!', 'patatras!' (comme par exemple dans la phrase Ja poljubila ego s pervogo vzgljada... Vzgljanula, a menja myšelovka xlop! 'Ce fut un coup de foudre... Je l'ai regardé et - pan! - je suis tombée dans la sourcière'), šast' (comme par

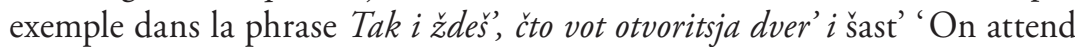
toujours que la porte s'ouvre et voilà [il entre]'), trax 'patatras!' (comme dans la phrase Axilla menja za sjurtuk. Ja povernulsja... trax! pola k čertu 'Achilla m’a attrapé par la redingote. Je me suis retourné et - patatras! - un pan est foutu'), f' ju (comme par exemple dans la phrase Imenie v stepi bylo - f'ju! 'On avait une propriété dans la steppe et - on ne l'a plus') ${ }^{42}$.

Néanmoins, dans les trois grammaires académiques, la distinction de deux groupes d'interjections seulement correspond, paradoxalement, à la définition de l'interjection proposée par Vinogradov lui-même: d'après lui, les interjections sont les signes qui servent à exprimer les réactions émotionnelles et "volontaires" face à la réalité de celui qui parle ${ }^{43}$. Si ni Fortunatov, ni Ščerba ne divisaient les interjections en plusieurs groupes de façon explicite, Vinogradov pouvait certainement s'appuyer sur la distinction des interjections chez A.A. Šaxmatov. Šaxmatov divisait les interjections en trois groupes:

4I. Ibid., p. 622.

42. Ibid. Soulignons en même temps qu'une petite différence, dans l'étude des interjections, entre les travaux de Vinogradov de 1938 et de 1947, consiste dans le fait que, dans le premier livre, Vinogradov mentionne neuf (et non dix) "classes d'interjections» [klassy meždometij]. Pourtant, en réalité, déjà en 1938, dix classes d'interjections sont distinguées: Vinogradov souligne que les mots qui se trouvent à la périphérie des interjections, entre les interjections et les verbes, font partie de cette dixième classe.

43. V.V. Vinogradov, Russkij jazyk, p. 611. 
1. les interjections qui expriment l'état d'agitation [vozbuždenie] de celui qui parle en général et, en particulier, les différents sentiments qui y sont liés, comme, par exemple, ax 'ah', $u x$ 'oh', gospodi, o gospodi 'Seigneur', 'ô Seigneur';

2. les interjections qui expriment certains sentiments particuliers [opredelennye čuvstva], comme da 'oui', fu 'fi', 'fi donc', 'pouah', ox 'oh', 'aïe', 'hélas', $t$ ' $u$ 'pouah', ču 'écoute', 'prête l'oreille';

3. les interjections qui expriment la volonté, comme na! 'tiens!', èj! 'holà!', 'ohé!', ajda!' allez!', pst!' viens ici', 'venez ici' (une interjection pour attirer l'attention de quelqu'un), allo! 'allô!', stop! ' halte!', 'stop!', tpru! 'hoo!', no!' hue!', brys', kyš 'ouste', tega-tega (les russophones appellent ainsi les oies), isi 'ici', 'viens ici' (pour appeler un chien), kuš 'couche!' (l'ordre adressé à un chien) ${ }^{44}$.

Or, dans la définition des interjections que Šaxmatov propose, on peut, comme chez Vinogradov, distinguer seulement deux caractéristiques de ces mots: les interjections expriment soit les sentiments (cf. les deux premiers groupes d'interjections qu'il distingue), soit la volonté de celui qui parle (cf. le dernier groupe) ${ }^{45}$.

Ainsi, même si dans la description des autres parties de la grammaire, la première grammaire académique (et donc les deux autres) avait été basée sur les théories grammaticales de Vinogradov, ce chercheur n'était pas le premier linguiste soviétique à distinguer les interjections en tant que groupe de mots à part, qui ne s'intègrent ni dans la catégorie des mots lexicaux, ni dans celle des mots grammaticaux. Si dans la description des autres parties de la grammaire russe Vinogradov avait réconcilié deux "traditions» différentes, il n'a pas dû le faire en étudiant les interjections, car les représentants des deux "traditions" avaient traité ces mots de la même façon. Quant à la distinction de précisément deux groupes d'interjections, les auteurs des grammaires académiques de la langue russe, en s'écartant de la division détaillée de Vinogradov, pouvaient en même temps s'appuyer sur la définition de l'interjection proposée par ce dernier.

Ekaterina Velmezova

Université de Lausanne

44. A.A. Šaxmatov, Sintaksis russkogo jazyka, p. 507.

45. Ibid. 


\section{BIBLIOGRAPHIE}

Barxudarov, Stepan Grigor'evič, Grammatika russkogo jazyka. Učebnik dlja 6-go i 7-go klassov semiletnej i srednej školy, Moskva, Gosudarstvennoe učebno-pedagogičeskoe izdatel'stvo Ministerstva prosveščenija RSFSR, 1947 (1939-1941), parties I-II. Bel'čınov, Julij Abramovič, Akademik V.V. Vinogradov (1895-1969). Tradicii $i$ novatorstvo $v$ nauke o russkom jazyke, Moskva, Vysšaja škola, 2004.

Boduèn de Kurtenè, Ivan Aleksandrovič (Baudoin de Courtenay, Jan Ignaci), "Lingvističeskie zametki i aforizmy» (1903), in I.A. Boduèn de Kurtenè, Izbrannye trudy, vol. II, p. 33-55.

—, «Jazyk i jazyki» (1904), in I.A. Boduèn de Kurtenè, Izbrannye trudy, vol. II, p. 67-95.

-, Izbrannye trudy po obščemu jazykoznaniju, Moskva, Izdatel'stvo Akademii nauk SSSR, 1963, vol. I-II.

Fortunatov, Filipp Fedorovič, Sravnitel'noe jazykovedenie. Obščij kurs (1901-1902), in F.F. Fortunatov, Izbrannye trudy, Moskva, Gosudarstvennoe učebno-pedagogičeskoe izdatel'stvo Ministerstva prosveščenija RSFSR, 1956-1957, vol. I, p. 23-197.

Gvozdev, Aleksandr Nikolaevič, Sovremennyj russkij literaturnyj jazyk, Moskva, Prosveščenie, 1973 (1955-1956), vol. I.

Ivanova, Irina Petrovna, «O polevoj strukture častej reči v anglijskom jazyke", in Teorija jazyka, metody ego issledovanija i prepodavanija. K 100-letiju so dnja roždenija L'va Vladimiroviča Ščerby, otv.red./éd. Ruben Ivanovič Avanesov, Leningrad, Nauka, 1981, p. $125-129$.

JarošEvskij, Mixail Grigor'evič, «Ponjatie vnutrennej formy u Potebni», Izvestija Akademii nauk SSSR, Serija literatury i jazyka, V/5 (1946), p. 335-399.

Jespersen, Otto, La philosophie de la grammaire, Paris, Les Editions de Minuit, 1971 (1924). 
Kručinina, Irina Nikolaevna, "Meždometija», in Lingvističeskij ènciklopedičeskij slovar', otv.red./éd. Viktorija Nikolaevna Jarceva, Moskva, Sovetskaja ènciklopedija, 1990, p. 290-291.

"Meždometie", in Bol'šaja sovetskaja ènciklopedija (troisième édition), gl.red./dir. Aleksandr Mixajlovič Proxorov, Moskva, Sovetskaja ènciklopedija, 1974, vol. XV, p. 591.

Pospelov, Nikolaj Semenovič, Učenie o častjax reči v russkoj grammatičeskoj tradicii. Materialy k kursam jazykoznanija, Moskva, Izdatel'stvo Moskovskogo universiteta, 1954.

ŠAхматоv, Aleksej Aleksandrovič, Sintaksis russkogo jazyka, The Hague, Mouton \& Co, 1963 (1925-1927).

Ščerba, Lev Vladimirovič, "O “diffuznyx zvukax” (1935), in Sumerki lingvistiki. Iz istorii otečestvennogo jazykoznanija. Antologija, otv. red./éd. Vladimir Petrovič Neroznak, Moskva, Academia, 2001, p. 360-362.

—, "O častjax reči v russkom jazyke» (1928), in L.V. Ščerba, Jazykovaja sistema i rečevaja dejatel'nost', Moskva, URSS, 2004, p. 77-100.

Švedova, Natal'ja Jul'evna, «Meždometija kak grammatičeski značimyj èlement predloženija v russkoj razgovornoj reči ", Voprosy jazykoznanija, 1 (1957), p. 85-95.

-, (otv.red./éd.), Grammatika sovremennogo russkogo literaturnogo jazyka, Moskva, Nauka, 1970.

—, "Meždometija", in Russkij jazyk. Ėnciklopedija, otv.red./éd. Fedot Petrovič Filin, Moskva, Sovetskaja ènciklopedija, 1979, p. 137.

Švedova, Natal'ja Jul'evna, Arutjunova, Nina Davidovna, Bondarko, Aleksandr Vladimirovič, Ivanov, Valerij Vasil'evič, Lopatin, Vladimir Vladimirovič, Uluxanov, Igor' Stepanovič, Filin, Fedot Petrovič (otv.red./éds), Russkaja grammatika, Moskva, Nauka, 1980, vol. I-II.

VAndries, Žozef (Vendryes, Joseph), Jazyk. Lingvističeskoe vvedenie $v$ istoriju [Le langage. Introduction linguistique à l'histoire], tr. inconnu (probablement, Mixail Andreevič Solonino - E. V.), Moskva, Socèkgiz, 1937 (1923).

Velmezova, Ekaterina, "La sémantique idéologique entre Marr et Staline», Cahiers de l'ILSL, 17 (2004), p. 315-335.

-, Les lois du sens: la sémantique marriste, Berne, ("Slavica Helvetica»), Peter Lang, 2007. 
—, «Eléments primaires du langage humain ou catégories psycholinguistiques propres aux langues? Les interjections chez L.V. Ščerba", Cahiers de l'ILSL, 24 (2008), p. 211-222.

-, "L'interjection dans la linguistique russe du XXe siècle: entre langue(s) et langage", in Contributions suisses au XIVe congrès mondial des slavistes à Ohrid, septembre 2008, éd. Patrick Sériot, Berne, ("Slavica Helvetica»), Peter Lang, 2008, p. 303-325.

—, «Da i net: časticy ili meždometija? Iz istorii akademičeskix grammatik prošlogo veka", in Sbornik statej učastnikov Meždunarodnoj konferencii "Assercija i negacija" (Moskva, maj 2007), otv.red./éd. Nina Davidovna Arutjunova, Moskva, Indrik, 2009 (à paraître).

Vendryes, Joseph, Le langage. Introduction linguistique à l'histoire, Paris, Editions Albin Michel, 1968 (1923).

Vinogradov, Viktor Vladimirovič, Sovremennyj russkij jazyk, Moskva, Gosudarstvennoe učebno-pedagogičeskoe izdatel'stvo Narkomprosa RSFSR, 1938, vol. I (Vvedenie v grammatičeskoe učenie o slove) - II (Grammatičeskoe učenie o slove).

-, "Učenie akad. A.A. Šaxmatova o grammatičeskix formax slov i o častjax reči v sovremennom russkom jazyke», in [Aleksej Aleksandrovič Šaxmatov,] Iz trudov A.A. Šaxmatova po sovremennomu russkomu jazyku (učenie o častjax reči), Moskva, Gosudarstvennoe učebno-pedagogičeskoe izdatel'stvo Ministerstva prosveščenija RSFSR, 1952, p. 3-26.

-, Russkij jazyk. Grammatičeskoe učenie o slove, Moskva, Vysšaja škola, 1986 (1947).

Vinogradov, Viktor Vladimirovič, Istrina, Evgenija Samsonovna, Barxudarov, Stepan Grigor'evič (otv.red./éds), Grammatika russkogo jazyka, Moskva, Izdatel'stvo Akademii nauk SSSR, 19521954, vol. I-II.

- , (otv.red./éds), Grammatika russkogo jazyka, Moskva, Izdatel'stvo Akademii nauk SSSR, 1960, vol. I-II.

Zinder, Lev Rafailovič, Maslov Jurij Sergeevič, L.V. Ščerba - lingvistteoretik i pedagog, Leningrad, Nauka, 1982. 
